Check for updates

Cite this: RSC Adv., 2017, 7, 24022

Received 15th February 2017

Accepted 20th April 2017

DOI: 10.1039/c7ra01914a

rsc.li/rsc-advances

\title{
Agitating transformation during vinyl chloride suspension polymerization: aggregation morphology and PVC properties
}

\begin{abstract}
Rui Guo, ${ }^{a}$ Erlei Yu, ${ }^{\text {b Jia Liu }}{ }^{a}$ and Zhong Wei (D) *ab
In this study, the effect of agitation rate on the aggregation morphology of the suspension poly(vinyl chloride) (PVC) grains, especially their primary particles, at different conversions was investigated. The results of scanning electron microscopy and laser particle size analysis indicated that the size and particle size distribution (PSD) of the final resin grains were mainly affected by the agitation rate, which were obtained before the monomer conversion reached $27 \%$. A high agitation rate was beneficial to obtain PVC resin with small grains and narrow PSD. However, higher agitation rate after $27 \%$ conversion would lead to more aggregation and fusion of the primary particles and make the primary particles larger. The resins produced by a variable speed process (from $600 \mathrm{rpm}$ to $300 \mathrm{rpm}$ when the grains were basically formed, i.e., $27 \%$ conversion) displayed better aggregation morphology and processability compared to those produced by the simpler high or low agitation rate method. Moreover, the relationship between the aggregation morphology and the macroscopic properties of the PVC resin was established.
\end{abstract}

\section{Introduction}

Poly(vinyl chloride) (PVC) is one of the four general plastic materials and widely applied in many fields. ${ }^{1}$ Almost $80 \%$ of PVC is produced via suspension polymerization (S-PVC), ${ }^{2-8}$ which offers many advantages including production of pure material and easy control of the reaction heat loss. The essential feature of suspension polymerization is the bulk polymerization of a large number of small droplets dispersed in aqueous media. Vinyl chloride monomer (VCM) is dispersed into small droplets in water via dispersion and stirring. The reaction starts with the thermal decomposition of the initiator into primary radicals. Since PVC has low solubility in VCM, the polymer chains are precipitated out of the monomer phase at considerably low conversion. ${ }^{9,10}$ Finally, the polymer chains form primary particles after nucleation, growth, aggregation, and fusion in the monomer droplet.

The aggregation morphology of the PVC grains is important for the processing characteristics of the PVC resin, ${ }^{11}$ cold plasticizer absorption (CPA) of the final resin, and removal of the residual monomers at the end of polymerization. ${ }^{12-14}$ Therefore,

${ }^{a}$ Key Laboratory for Green Processing of Chemical Engineering of Xinjiang Bingtuan, School of Chemistry and Chemical Engineering, Shihezi University, Shihezi, China. E-mail: steven_weiz@sina.com

${ }^{b}$ Key Laboratory of Materials-Oriented Chemical Engineering of Xinjiang Uygur Autonomous Region, Engineering Research Center of Materials-Oriented Chemical Engineering of Xinjiang Bingtuan, School of Chemistry and Chemical Engineering, Shihezi University, Shihezi, China investigating the aggregation morphology of PVC grains is considerable work.

The dynamic balance between droplet coalescence and breakage processes is largely affected by agitation (i.e., agitation type and agitation rate) during monomer dispersion. Thus, agitation is one of the most important factors that determines the morphology of S-PVC, including the mean grain size and the particle size distribution (PSD). Barclay found that the macroscopic properties of S-PVC grains, such as size and internal voids, are largely controlled by a balance among interfacial tension, colloid stability, and agitation..$^{15}$ The effect of low agitation rate in the range of 250-400 rpm on the porosity of the final PVC resin was reported by Visentini who found that porosity increased with the increasing agitation rate. ${ }^{16}$ Davidson studied quiescent polymerization and polymerization with agitation. The results showed that the coagulation of primary particles could be completed early depending on the agitation conditions, and the formation of a three-dimensional network might be substantially advanced. Primary particles tend to grow as individual particles, and their aggregation is delayed when they are polymerized under a quiescent condition. ${ }^{17}$ Alexopoulos proposed a model to predict the PSD of primary particles and found that agitation rate is a remarkably important variable.$^{18}$ However, Smallwood reported that when the range of agitation rate is considerably narrow (175-225 rpm), it exerts no obvious effect on the size of the primary particles. ${ }^{14}$

Although the effect of agitation rate on the morphology of the final S-PVC grains has been researched in the past few decades, most of these studies were focused on the outer morphology of the PVC grains. By contrast, the study on primary 
particles is only limited to a narrow range of agitation rate. The effect of the growth, aggregation, and fusion of primary particles on the entire polymerization process remains unclear. In this study, to more comprehensively investigate the relationship between the aggregation morphology (grains, primary particles) and agitation and produce PVC resins with better properties, we studied the growth, aggregation, and fusion of primary particles over relatively wide agitation rates (300-600 rpm) at different conversions. The formation of grain skin was also investigated.

\section{Experimental}

\subsection{Materials}

VCM and demineralized water were supplied by the Xinjiang Tianye (Group) Co., Ltd. (Xinjiang, China). Hydroxypropyl methylcellulose (HPMC, 65RT50), which served as a dispersant, was purchased from Ruitai Cellulose Co., Ltd. (Shandong, China). Poly(vinyl alcohol) (PVA) with the hydrolysis degrees of 69-72\% (PVA1) and 78.5-81.8\% (PVA2) was supplied by the Tai Chang Resin Co., Ltd. (Taiwan, China). Di-(2-ethylhexyl) peroxydicarbonate (W-60) was used as the initiator and obtained from the Tianjin Akzo Noble Chemical Corporation (Tianjin, China). Boric acid was supplied by the Tianjin North Union Fine Chemicals Development Co., Ltd. (Tianjin, China). Ammonium bicarbonate, a buffer reagent, was provided by the Chinese Medicine Group Chemical Reagent Co., Ltd. (Shanghai, China). Iodine was provided by the Tianjin Yongsheng Fine Chemical Co., Ltd. (Tianjin, China). Potassium iodide was supplied by the Tianjin Sailboat Chemical Reagent Technology Co., Ltd. (Tianjin, China). Dioctyl phthalate (DOP) was supplied by the Tianjin Fu Yu Fine Chemical Co., Ltd. (Tianjin, China) and used as a plasticizer without any purification.

\subsection{Polymerization}

A 5 L stainless-steel autoclave jacketed vessel with two flat-blade impellers and two anchor paddles was used for VCM suspension polymerization. The reactor temperature was automatically controlled through an ABB control system. First, a certain amount of demineralized water, compound dispersant $(0.12$ wt $\%$ in proportion to the amount of VCM, HPMC : PVA1 : PVA2 $=10: 7: 7 \mathrm{wt} \%), 1 \mathrm{~g}$ of ammonium bicarbonate buffer salt solution, $0.8 \mathrm{~g}$ of initiator, and the remaining demineralized water were added into the autoclave in sequence. The total amount of demineralized water was $3 \mathrm{~kg}$. Subsequently, the autoclave was closed, evacuated, purged with nitrogen, and then evacuated again to reduce the concentration of residual oxygen. After this, $1 \mathrm{~kg}$ of VCM was precisely injected into the reactor through the feed valve. Finally, agitation was initiated at $300 \mathrm{rpm}$ or $600 \mathrm{rpm}$. The reaction was carried out at a constant temperature $\left(57.5^{\circ} \mathrm{C}\right)$ after $30 \mathrm{~min}$ of cold stirring. The starting time of the reaction was obtained when the internal temperature of the reactor reached $57.5{ }^{\circ} \mathrm{C}$. After a certain time, polymerization was terminated and the residual monomer was evacuated. The prepared resins were filtered and washed with demineralized water, centrifuged, and dried at $50{ }^{\circ} \mathrm{C}$ for $36 \mathrm{~h}$.
Table 1 Resin samples polymerized under different conditions

\begin{tabular}{|c|c|c|c|c|}
\hline \multirow{2}{*}{$\begin{array}{l}\text { Polymerization } \\
\text { time/min }\end{array}$} & \multicolumn{2}{|l|}{$300 \mathrm{rpm}$} & \multicolumn{2}{|l|}{$600 \mathrm{rpm}$} \\
\hline & Sample & Conversion/\% & Sample & Conversion $/ \%$ \\
\hline 8 & L-1.1\% & 1.1 & $\mathrm{H}-0.7 \%$ & 0.7 \\
\hline 15 & L-1.9\% & 1.9 & H-1.5\% & 1.5 \\
\hline 30 & L-5.9\% & 5.9 & H-4.5\% & 4.5 \\
\hline 45 & L-6.1\% & 6.1 & $\mathrm{H}-7.0 \%$ & 7.0 \\
\hline 60 & L-11.8\% & 11.8 & $\mathrm{H}-13.8 \%$ & 13.8 \\
\hline 90 & L-17.3\% & 17.3 & $\mathrm{H}-22.0 \%$ & 22.0 \\
\hline 120 & L-27.2\% & 27.2 & H-28.5\% & 28.5 \\
\hline 180 & L-42.0\% & 42.0 & $\mathrm{H}-45.5 \%$ & 45.5 \\
\hline 240 & L-59.4\% & 59.4 & H-59.6\% & 59.6 \\
\hline 300 & L-66.5\% & 66.5 & $\mathrm{H}-70.3 \%$ & 70.3 \\
\hline 360 & L-70.0\% & 70.0 & $\mathrm{H}-73.3 \%$ & 73.3 \\
\hline 420 & L-79.5\% & 79.5 & $\mathrm{H}-77.5 \%$ & 77.5 \\
\hline 480 & L-82.5\% & 82.5 & H-80.5\% & 80.5 \\
\hline 600 & L-85.7\% & 85.7 & H-83.0\% & 83.0 \\
\hline
\end{tabular}

The resin samples obtained after different reaction times and with different conversions are listed in Table 1.

\subsection{Characterization}

2.3.1 Monomer conversion. Monomer conversion was determined by gravimetry:

$$
\text { Monomer conversion }=\frac{m_{\mathrm{pvc}}}{m_{\mathrm{monomer}}} \times 100 \%
$$

where $m_{\mathrm{pvc}}$ is the quality of PVC and $m_{\text {monomer }}$ is the quality of the original added VCM.

2.3.2 Scanning electron microscopy. The aggregation morphology of the interior and exterior of the PVC grains was observed using a scanning electron microscope (SEM, JSM6490LV, Jeol, Tokyo, Japan). An appropriate number of sample grains were fixed in conductive adhesive and coated with a thin layer of gold using a gold sputter coater in vacuum. Subsequently, the aggregation morphology of the PVC grains was observed under a SEM. The samples were used to observe the interior aggregation morphology, especially, after mechanical breakdown in the presence of liquid nitrogen.

2.3.3 Grain size and PSD. The grain size and PSD of the samples were measured using a Microtrac Model S3500 Laser Particle Size Analyzer (Florida, US). The mean size of the primary particles (Sauter mean diameter D32) was measured using the method reported by Darvishi. ${ }^{5}$

2.3.4 Porosity and bulk density (BD). The porosity of the sample grains was characterized by cold plasticizer absorption. Herein, $0.1 \mathrm{~g}$ of absorbent cotton was precisely weighed in a centrifuge tube. A weighed amount of sample resin was placed in a cotton tube, and $2 \mathrm{~mL}$ of DOP was absorbed for $15 \mathrm{~min}$ at room temperature $\left(20 \pm 2{ }^{\circ} \mathrm{C}\right)$. The unabsorbed DOP was removed by centrifugal separation (3000 rpm, $60 \mathrm{~min})$. The BD of the samples was measured using the Chinese standard GB/T 5761-2006 method.

2.3.5 PVA concentration. The PVA concentration in the mother liquor was measured via the UV-Vis method reported by 
Zerfa. ${ }^{\mathbf{1 9 2 0}}$ The general principle of this method is that PVA forms a green complex with iodine in the presence of boric acid, which is absorbed in the range of $650-690 \mathrm{~nm}$.

2.3.6 Processability. The processability of the resin was tested using a HAAKE rheometer (PolyLab QC, Germany). Typically, $60 \mathrm{~g}$ of PVC was mixed with $1.8 \mathrm{~g}$ of organic tin and $0.48 \mathrm{~g}$ of paraffin wax. The mixture was measured under the following conditions: temperature of mixing head, $180^{\circ} \mathrm{C}$; rotor speed, $35 \mathrm{rpm}$.

\section{Results and discussion}

\subsection{Effect of different agitation rates on the relationship between conversion and reaction time}

As can be seen in Fig. 1, the results showed that the agitation rate exerted no significant influence on the polymerization rate. H. G. Yuan also mentioned that agitation conditions seem to have no influence on the polymerization rate in the batch suspension homopolymerization processes. ${ }^{21}$ The reaction rate of vinyl chloride suspension polymerization mainly depended on temperature ${ }^{22}$ and the initiator system. ${ }^{2}$ In this study, the polymerization was carried out in a $5 \mathrm{~L}$ autoclave. The relatively small reactor volume and high agitation rate $(300-600 \mathrm{rpm})$ made the heat transfer steady. Therefore, there was no significant difference in conversion with respect to different agitation rates.

\subsection{Effect of different agitation rates on PVC resin grains}

To investigate the formation process of S-PVC grains and the influence of the agitation rate on this process, different sample grains polymerized with different conversions at different agitation rates were imaged using SEM (Fig. 2). The images indicate that the macroscopic morphology of the PVC grains at different conversions gradually changed from rod-like grains to spheroidal grains at both stirring speeds. At a low monomer conversion $(<17-27 \%)$, a large amount of residual monomers were present and a few primary particles formed in the monomer droplets. In addition, the grain's pericellular membrane

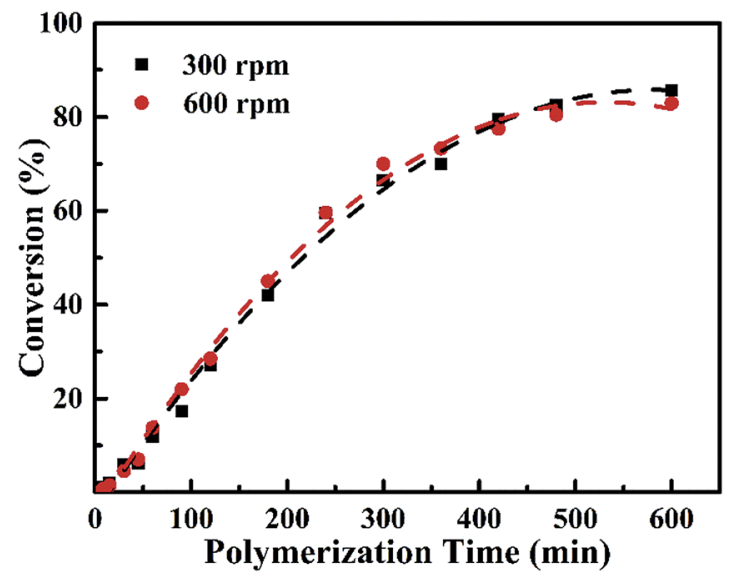

Fig. 1 Monomer conversion change with reaction time at different agitation rates.

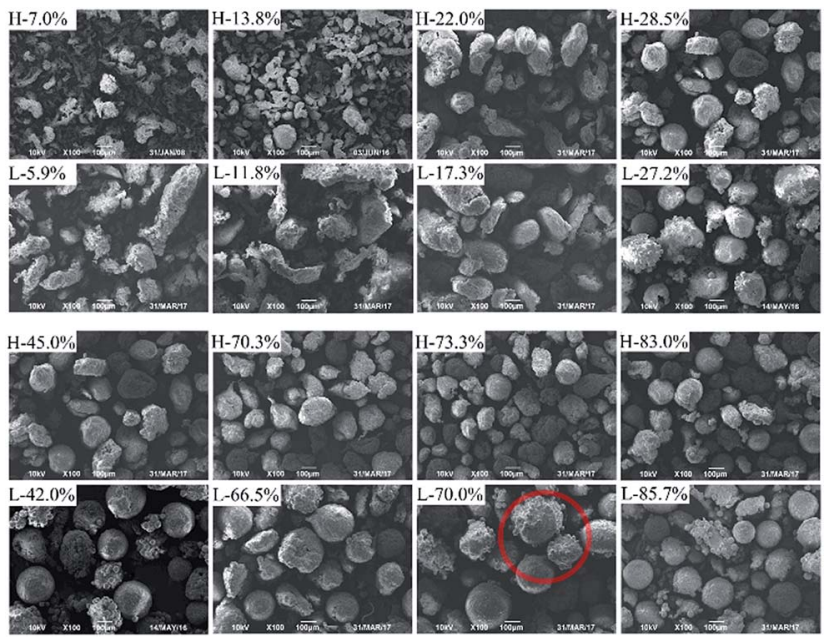

Fig. 2 Scanning electron microscopy (SEM) images of PVC grains.

(skin) was not sufficiently dense. All the abovementioned phenomena caused the droplets to contract and deform when the residual monomers were removed. This effect may explain the rod-like morphology of the grains. The amount of residual monomers decreased with the increase in conversion. The aggregation of primary particles progressively formed a type of inner skeleton (three-dimensional network structure) inside the monomer droplets, ${ }^{5}$ and the skin became increasingly tough (Fig. 5). Consequently, the droplets were prevented from excessive contraction, and then, the PVC grains became approximately spherical.

A comparison of the images shows that resin grains polymerized at a low agitation rate contained more multicellular grains. Monomer droplets were gradually transformed into viscous monomer-polymer droplets (or latex particles) whose coalescence formed multicellular grains, as can be seen in Fig. $2 .^{23}$ These viscous droplets may have difficulty in coalescing at $600 \mathrm{rpm}$, which thereby reduced the number of multicellular grains.

The PVC grains evolved from monomer droplets, ${ }^{24}$ and the size and uniformity of the droplets had a great influence on the grain size and PSD. The distribution of the monomer droplets

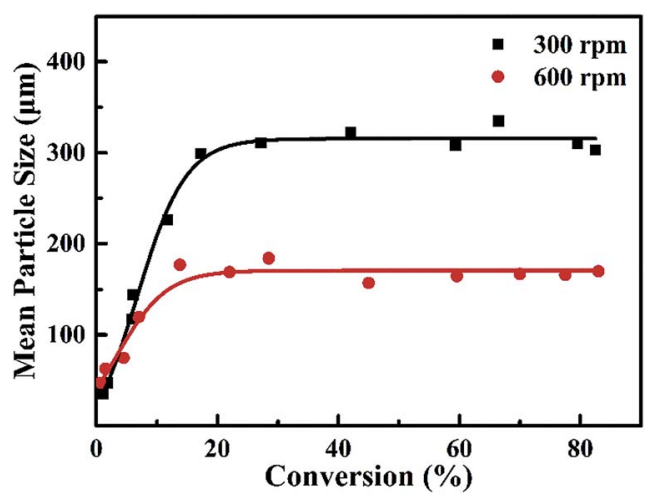

Fig. 3 Grain size of PVC resins polymerized at different agitation rates with different conversions. 


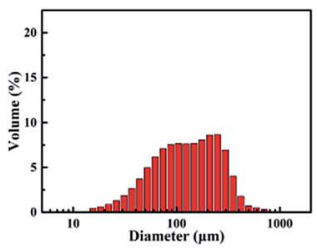

$\mathrm{H}-7.0 \%$

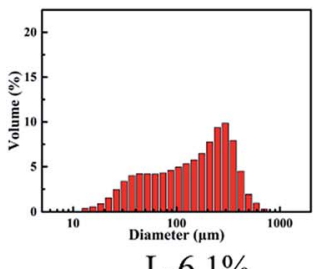

L-6.1\%

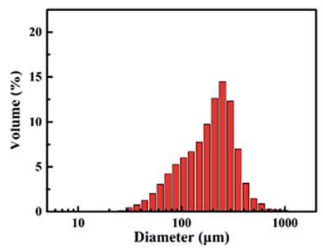

H-13.8\%

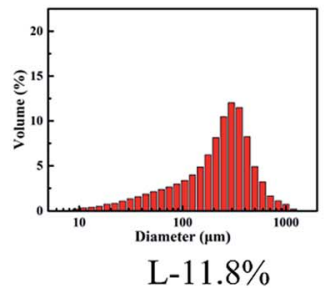

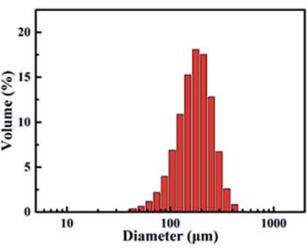

H- $28.5 \%$

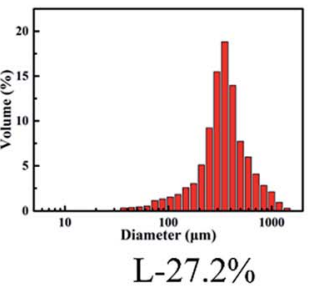

L-27.2\%

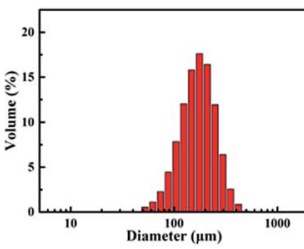

$\mathrm{H}-59.6 \%$

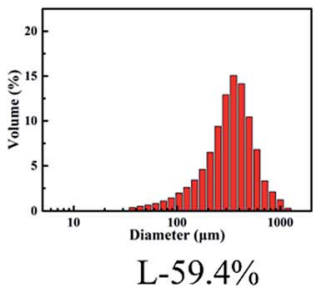

L-59.4\%

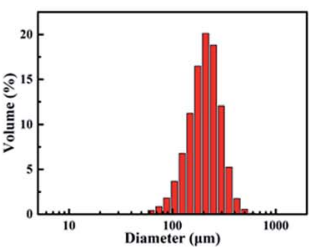

$\mathrm{H}-83.0 \%$

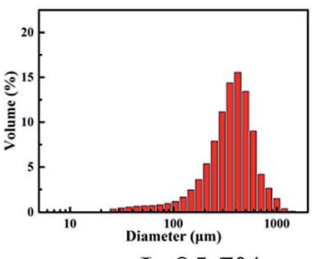

L-85.7\%

Fig. 4 Particle size distribution of PVC grains.

was much smaller and became more uniform during polymerization at high stirring speeds. ${ }^{19}$ The mean grain size of PVC significantly decreased with the increasing agitation rate (300 rpm, about $320 \mu \mathrm{m} ; 600 \mathrm{rpm}$, about $165 \mu \mathrm{m}$ ), as shown in Fig. 3. Furthermore, the grain size increased with conversion and then remained constant when the conversion reached $X_{\mathrm{b}}$ (a conversion at which grains were basically formed and the size and PSD of the grains showed no obvious change after that). Herein, two reasons can explain this phenomenon. First, from the aspect of individual grains, a three-dimensional network structure was initially formed over the conversion range of 10$17 \%$ and completely formed at about $27 \%$. This result was consistent with the experimental result of Smallwood. ${ }^{\mathbf{1 4}}$ Moreover, this structure could prevent droplet contraction when the residual monomer was removed. As a result, the individual grain size increased with monomer conversion, and then, the grain size was almost invariant when the conversion was beyond $27 \%$. From the aspect of multicellular grains, monomer droplets became gradually viscous with the increasing conversion, which led to a coalescence of droplets and then formed the multicellular grains. Consequently, the grain size increased. However, when the conversion reached about $27 \%$, the skin became remarkably dense (Fig. 5) and the droplets could not aggregate. As a result, the number of multicellular grains no longer increased with conversion, and then, the grain size became constant after $27 \%$ conversion.

Additionally, the $X_{\mathrm{b}}$ of polymerization at high agitation rate was lower, as shown in Fig. 3 (low: 300 rpm, about 27\%; high: $600 \mathrm{rpm}$, about $22 \%$ ). This phenomenon is due to the increase in the breakage rate of droplets and the coalescence rate at high agitation speeds. ${ }^{19}$ Consequently, the inner skeleton inside the polymerizing monomer droplets and the skin formed earlier (as shown in Fig. 5). Therefore, the ability of droplets to resist shrinkage was stronger at a high agitation rate than that of the droplets polymerized at a low agitation rate.

As shown in Fig 4, the PSD of the grains became narrow with the increase in conversion and then had no obvious change after about $27 \%$ conversion. This was consistent with the formation of the grain. Moreover, the PSD changed from wide to narrow with the increasing agitation rate, and the grain size of the corresponding peak became much smaller. The monomer droplets showed a much narrower size distribution and were more uniform at a high stirring speed. ${ }^{19}$ Therefore, the PVC grains became smaller and more uniform compared to the samples polymerized at low stirring speeds.

\subsection{Effect of different agitation rates on the formation of pericellular membrane (skin)}

Fig. 5 presents the evolution of the skin with conversion at various agitation rates. The porosity of the skin obviously decreased with the increase in conversion at the two agitation rates and disappeared at about $30-40 \%$ conversion. Given the small amount of VCM dissolved in water and the poor solubility of PVC in water, PVC oligoradicals were precipitated. After this,
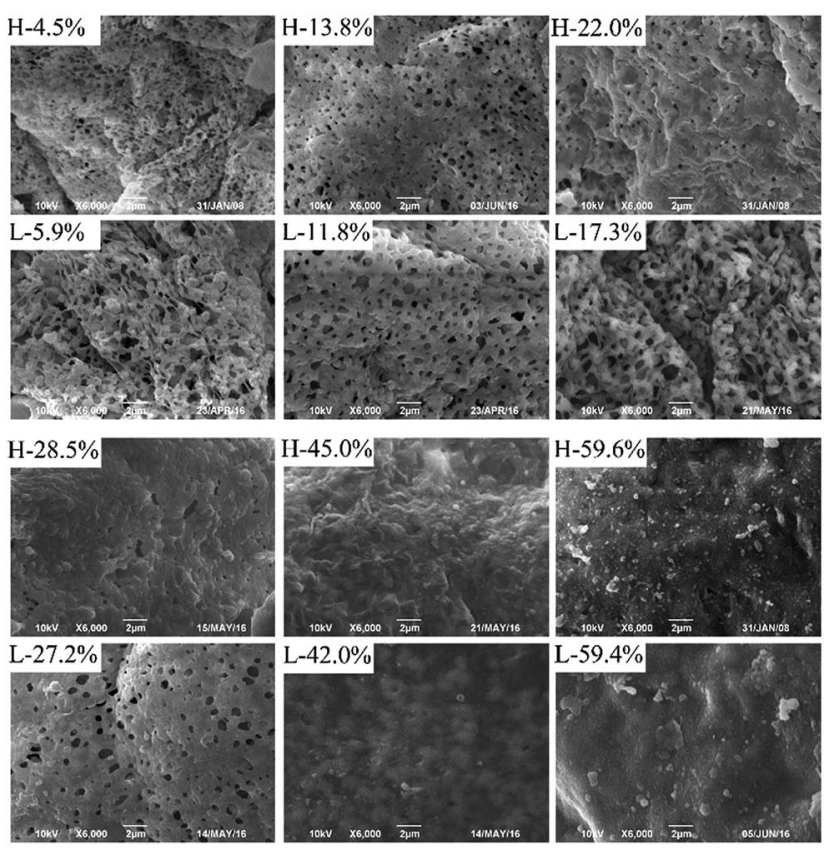

Fig. 5 SEM images of the grain skin. 


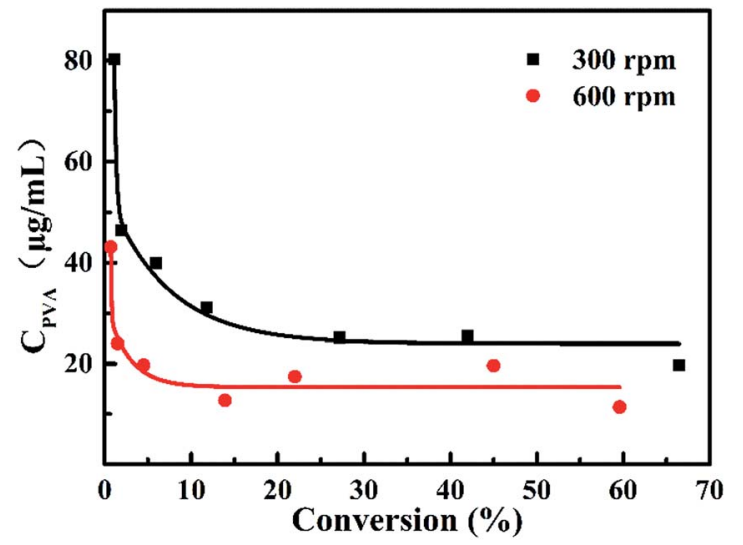

Fig. 6 Concentration of PVA in the mother liquor polymerized at different agitation rates and conversions.

some of these oligoradicals migrated into the polymer-rich phase and continued to grow at the primary stage of polymerization. However, at a relatively high conversion, the oligoradicals could not migrate into the droplets. ${ }^{25}$ They gradually aggregated to form primary particles at the surface of the droplets and became part of the skin. Furthermore, the primary particle nuclei and primary particles moved towards the inner surface of the droplets in the presence of a centrifugal force, which lowered the porosity of the skin..$^{23,26}$ Moreover, according to Fig. 6, it was concluded that PVA in the aqueous medium was gradually absorbed on the surface of the droplets at about $27 \%$ conversion and formed the skin of the PVA- $g$-PVC copolymer. ${ }^{17,24,27,28}$ Consequently, the porosity of the skin became smaller.

Note than the porosity of the skin polymerized at $600 \mathrm{rpm}$ decreased faster than that of the samples polymerized at

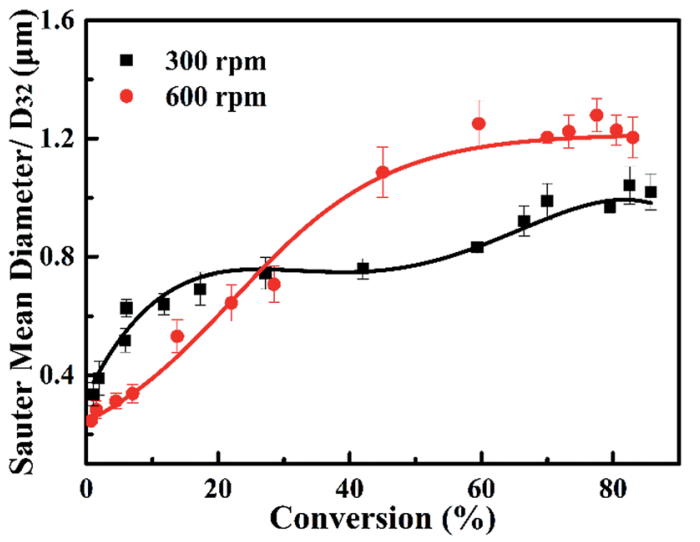

Fig. 8 Size of the primary particles polymerized at different agitation rates with different conversions.

$300 \mathrm{rpm}$ (i.e., the skin of PVC grains formed early when polymerized at a high agitation rate). This observation was mainly explained by the fact that the breakage and coalescence rates of droplets increase at high agitation speeds, ${ }^{19}$ and this condition increased the opportunity of PVA in the droplet surface to come in contact with the radicals inside the droplets. Finally, the PVA$g$-PVC copolymer rapidly formed. Additionally, the primary particles easily moved towards the inner surface of the droplet at high agitation rates such that the porosity of the skin quickly decreased. $^{23}$

\subsection{Effect of different agitation rates on the growth and aggregation of primary particles}

In general, the size of the primary particles increased with the increase in conversion because of growth, aggregation, and

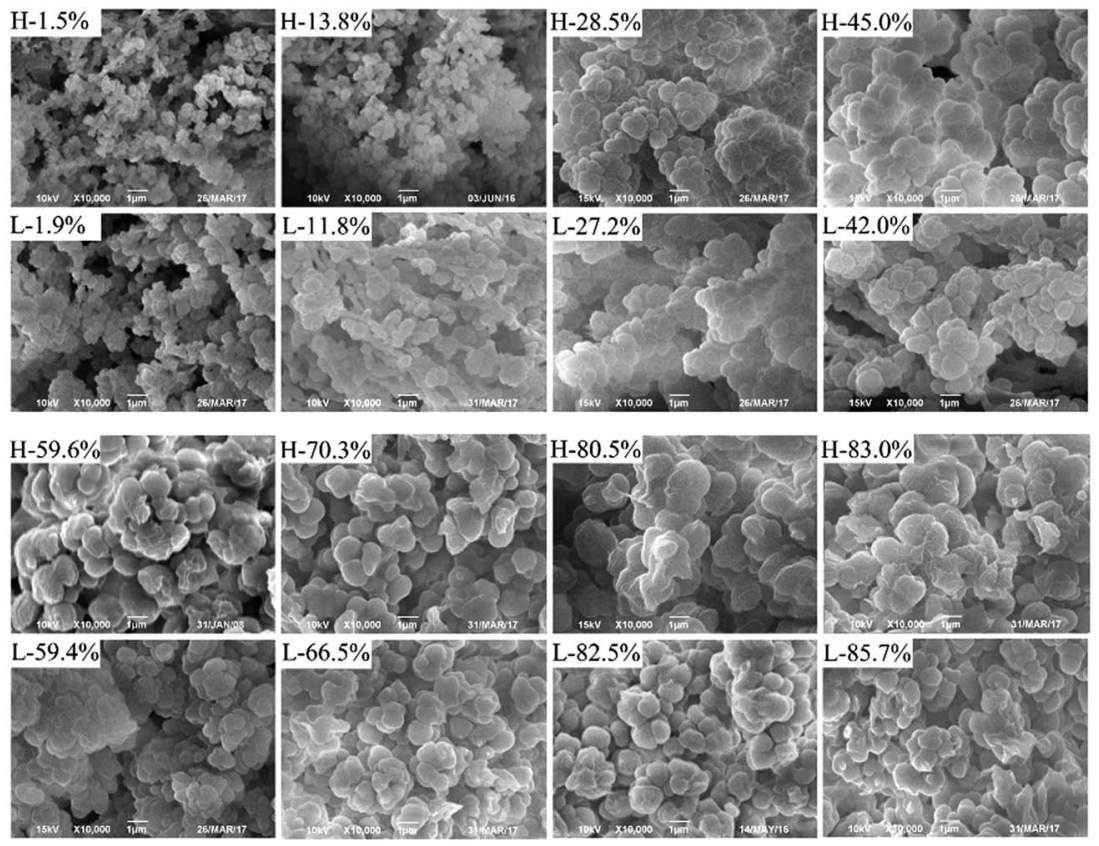

Fig. 7 SEM images of primary particles. 
fusion, as shown in Fig. 7 and 8. Furthermore, the primary particles polymerized at $600 \mathrm{rpm}$ were smaller than those polymerized at $300 \mathrm{rpm}$ when monomer conversion was less than $27 \%$. Because of the shear-induced flocculation, ${ }^{\mathbf{1 4}}$ the primary particle nuclei more easily precipitated from the monomer phase at higher agitation rates. As the nuclei grew, more and smaller primary particles formed under the abovementioned conversion conditions as compared to those formed under lower agitation rate conditions. By contrast, when the monomer conversion was above $27 \%$, the viscosity of the latex particle and the size of the primary particles became big enough, which reinforced the collision and aggregation of the primary particles at higher agitation rates. The size and fusion degree of the primary particles polymerized at high agitation rates were relatively higher than those polymerized at lower agitation rates.

As can be seen in Fig. 8, compared to polymerization at $600 \mathrm{rpm}$, the primary particle size reached a plateau with the increase in conversion at $300 \mathrm{rpm}$. When the conversion reached about $27 \%$, the aggregation of the primary particles formed a three-dimensional network structure in the droplets, which prevented the primary particles from continuously growing. ${ }^{5}$ The aggregation of primary particles was gradually hindered, and the size of the primary particles slowly increased until the reaction reached a conversion of about $60 \%$ (the motionless point). At the conversion of motionless point, the droplets were occupied by primary particles and the VCM filled in the interstitial spaces among the primary particles. ${ }^{29}$ The interstitial space of the primary particles was filled with the new polymer, and the primary particles became increasingly fused. Finally, when the conversion reached the critical conversion of about $80 \%$, the VCM phase disappeared ${ }^{18}$ and the polymerization only occurred in the polymer phase. The size of the primary particles showed no evident change. However, no plateau was observed when polymerization was carried out at $600 \mathrm{rpm}$. It may be due to much greater collision and aggregation than the inhibitory effect of the three-dimensional network under a high agitation rate condition, especially when the conversion was above $27 \%$.

\subsection{Effect of different agitation rates on the BD and porosity of PVC grains}

As shown in Fig. 9, the BD and CPA of the resin polymerized at two different agitation rates showed similar trends. The BD increased with the increasing conversion and then remained steady when the conversion reached about 80\%, whereas CPA presented a completely opposite trend. The reason for this phenomenon can be attributed to the continuous growth and aggregation of the primary particles with the increasing conversion. Consequently, the interior porosity of the PVC grains gradually decreased. However, the size of the primary particles barely changed such that the porosity (characterized by CPA) showed no obvious change when the conversion was greater than the critical conversion of $80 \%$. At $600 \mathrm{rpm}$, the BD was lower but the CPA was higher than that of the resin polymerized at $300 \mathrm{rpm}$ when the conversion was less than $27 \%$. An opposite situation appeared when the conversion was above $27 \%$. This observation was mainly explained by the change in the aggregation morphology of the primary particles, which led to a significant change in the interior porosity at $27 \%$ conversion at two different agitation rates. The results were consistent

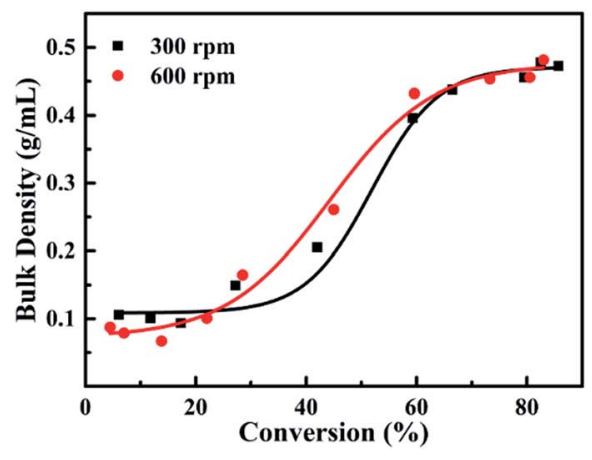

a

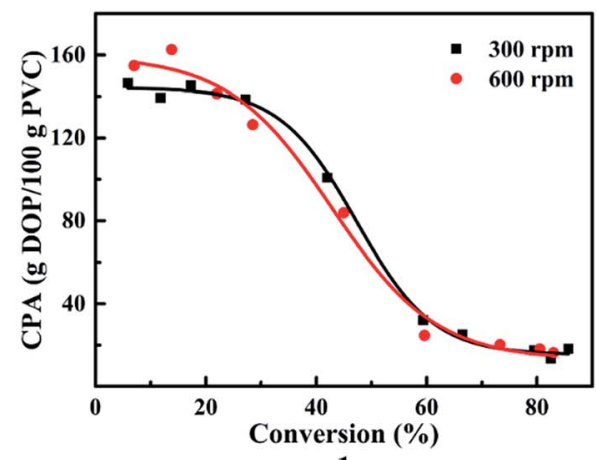

b

Fig. 9 The properties PVC resins polymerized at different agitation rates with different conversions: (a) bulk density and (b) cold plasticizer absorption.

Table 2 PVC resins polymerized using different types of agitation

\begin{tabular}{llllllll}
\hline Sample & $\begin{array}{l}\text { Agitation } \\
\text { speed/rpm }\end{array}$ & $\begin{array}{l}\text { Conversion/ } \\
\%\end{array}$ & $\begin{array}{l}\text { Grain size/ } \\
\mu \mathrm{m}\end{array}$ & $\begin{array}{l}\text { Primary particle } \\
\text { size/ } \mu \mathrm{m}\end{array}$ & $\begin{array}{l}\text { Bulk density/g } \\
\mathrm{mL}^{-1}\end{array}$ & $\begin{array}{l}\mathrm{CPA} / \mathrm{g} \text { DOP/100 g } \\
\text { pvc }\end{array}$ & $\begin{array}{l}\mathrm{plasticizing} \\
\text { time/min }\end{array}$ \\
\hline L-85.7\% & 300 & 85.7 & 340 & 1.019 & 0.472 & 16.98 & 1.182 \\
H-83.0\% & 600 & 83.0 & 170 & 1.203 & 0.481 & 16.72 & 0.955 \\
T-86.5\% & $600-(27 \%)-300$ & 86.5 & 165 & 1.035 & 0.482 & 17.70 & 0.883
\end{tabular}



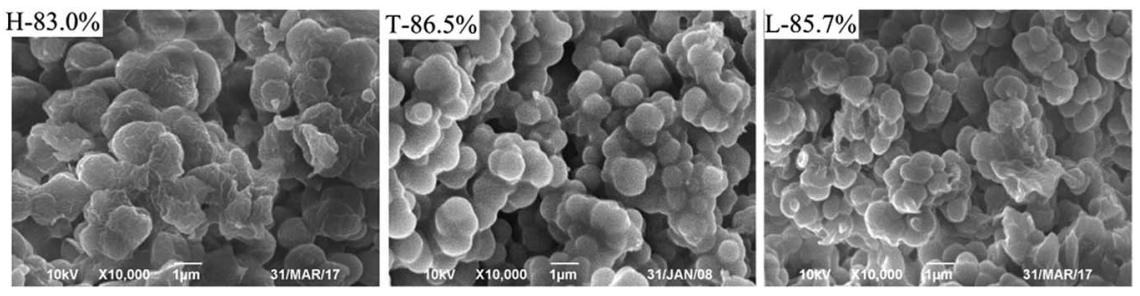

Fig. 10 SEM images of the primary particles.

with the tendency of the primary particle size, as shown in Fig. 8.

\subsection{Effect of variable speed on PVC grains}

For a more intuitive comparison and verification of the relationship between PVC grain aggregation morphology and the

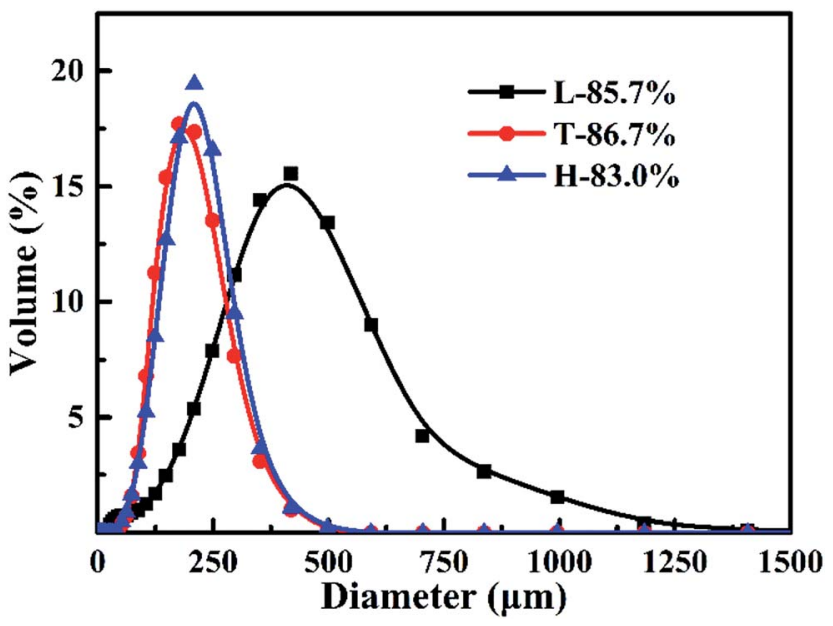

Fig. 11 Particle size distribution of different samples polymerized using different types of agitation.

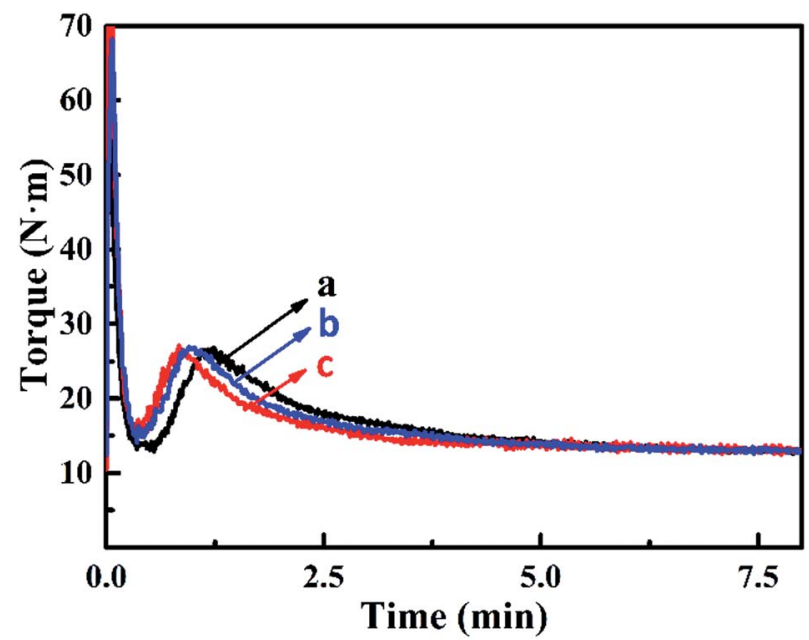

Fig. 12 Torque rheological curves of different samples: (a) L-85.7\%; (b) $\mathrm{H}-83.0 \%$; and (c) $\mathrm{T}-86.5 \%$. agitation condition, polymerization with variable speed (from $600 \mathrm{rpm}$ to $300 \mathrm{rpm}$ at $27 \%$ conversion) was also studied to compare with the simpler high or low agitation rate processes. The final monomer conversion was $86.5 \%$ and the sample was named T-86.5\%. The experimental results for the different agitation types are shown in Table 2 and Fig. 10-12. From the abovementioned studies, it was observed that when the conversion reached $27 \%$, the grains were formed; then, the grain size and PSD showed no obvious change. Moreover, when the variable speed process was adopted, the grain size and PSD of $\mathrm{T}-86.5 \%$ were similar to those at high agitation rate $\mathrm{H}-83.0 \%$ (Fig. 11). The sizes of these two particles were $165 \mu \mathrm{m}$ and 170 $\mu \mathrm{m}$, respectively. The final primary particle size mainly contributed to its growth, aggregation, and fusion. A high agitation rate was beneficial for the formation of smaller primary particles before $27 \%$ conversion was achieved, and a low agitation rate could restrain the aggregation and fusion of the primary particles after that. Therefore, the resin T-86.5\% prepared by the variable speed process displayed a similar primary particle size $(1.035 \mu \mathrm{m})$ to that prepared at a low agitation rate $\mathrm{L}-85.7 \%(1.019 \mu \mathrm{m})$, which was much smaller than that formed at the high agitation rate $\mathrm{H}-83.0 \%(1.203 \mu \mathrm{m})$. As a result, the aggregation morphology of the PVC grains was controlled through a variable speed operation, which produced resins, with a smaller size and narrower PSD of grains, and small-sized primary particles.

For investigating the properties, some resins properties such as $\mathrm{BD}, \mathrm{CPA}$, and plasticizing time of the three samples were characterized, as shown in Table 2 . The results showed that the $\mathrm{BD}$ of the three samples had no obvious change with respect to different agitation types. Compared to L-85.7\% (1.182 $\mathrm{min}$ ), the plasticizing time of $\mathrm{H}-83.0 \%$ and T-86.5\% decreased to $0.995 \mathrm{~min}$ and $0.883 \mathrm{~min}$, respectively. Because of the relatively narrow PSD and small grain size, the resin grains of these two samples could be more easily broken and then displayed better processing property. Furthermore, T-86.5\% had a higher CPA than $\mathrm{H}-83.0 \%$ with smaller primary particles and a lower fusion degree. ${ }^{30}$ Under the condition of similar PSD, it further reduced the plasticizing time. Therefore, the PVC resins obtained by variable speed operation, which have a narrow PSD and relatively small grains and primary particles, show a better processing property.

\section{Conclusions}

In summary, it was found that the agitation rates have a great influence on the aggregation morphology of S-PVC. When the 
conversion was less than about $27 \%$, it was beneficial to obtain PVC resin with small grains and narrow PSD and smaller and more primary particles at a high agitation rate. Then, a threedimensional network structure was formed and the size and PSD of the PVC grains showed no obvious change. When the conversion was over $27 \%$, a high agitation rate increased the aggregation and fusion degree of the primary particles and reduced the processability. Finally, via variable speed operation (from $600 \mathrm{rpm}$ to $300 \mathrm{rpm}$ ) at $27 \%$ conversion, we obtained PVC resins, with small particle size and narrow PSD, and small primary particles with better processing plasticization.

\section{Acknowledgements}

The financial support of the National Natural Science Foundation of China (No. 21464012), Program for Changjiang Scholars and Innovative Research Team in University (No. IRT_15R46), Funds for Distinguished Young Scientists of Xinjiang Bintuan (No. 2014CD001), and the Scientific Research Program of Shihezi University (No. RCZX201209) is gratefully acknowledged.

\section{Notes and references}

1 C. H. Chen, C. C. Teng and C. H. Yang, J. Polym. Sci., Part B: Polym. Phys., 2005, 43, 1465-1474.

2 Y. Saeki and T. Emura, Prog. Polym. Sci., 2002, 27, 2055-2131. 3 Y. Z. Bao and B. W. Brooks, J. Appl. Polym. Sci., 2003, 90, 954958.

4 N. Etesami, M. N. Esfahany and R. Bagheri, J. Appl. Polym. Sci., 2008, 110, 2748-2755.

5 R. Darvishi, M. Nasr Esfahany and R. Bagheri, J. Vinyl Addit. Technol., 2015, DOI: 10.1002/vnl.21501.

6 M. J. Bijhanmanesh and N. Etesami, J. Appl. Polym. Sci., 2016, 133, DOI: $10.1002 /$ app.44079.

7 A. F. Cebollada, M. J. Schmidt, J. N. Farber, N. J. Capiati and E. M. Vallés, J. Appl. Polym. Sci., 1989, 37, 145-166.

8 M. J. Bijhanmanesh, N. Etesami and M. N. Esfahany, J. Vinyl Addit. Technol., 2016, DOI: 10.1002/vnl.21534.
9 J. D. Cotman, M. F. Gonzalez and G. C. Claver, J. Polym. Sci., Part A-1: Polym. Chem., 1967, 5, 1137-1164.

10 D. G. Rance and E. L. Zichy, Pure Appl. Chem., 1981, 53, 377384.

11 C. L. Sieglaff, Pure Appl. Chem., 1981, 53, 509-520.

12 S. Ormondroyd, Br. Polym. J., 1988, 20, 353-359.

13 J. R. Defife, J. Vinyl Technol., 1980, 2, 95-99.

14 P. V. Smallwood, Polymer, 1986, 27, 1609-1618.

15 L. M. Barclay, Angew. Makromol. Chem., 1976, 52, 1-20.

16 A. Visentini, Plast., Rubber Compos., 1999, 28, 142-144.

17 J. A. Davidson and D. E. Witenhafer, J. Polym. Sci., Polym. Phys. Ed., 1980, 18, 51-69.

18 A. H. Alexopoulos and C. Kiparissides, Chem. Eng. Sci., 2007, 62, 3970-3983.

19 M. Zerfa, Vinyl chloride drop behaviour in suspension polymerisation reactors, Loughborough University, 1994.

20 M. Zerfa and B. W. Brooks, Colloids Surf., A, 1998, 132, 267273.

21 H. G. Yuan, G. Kalfas and W. H. Ray, J. Macromol. Sci., Part B: Phys., 1991, 31, 215-299.

22 C. Kiparissides, G. Daskalakis, D. S. A. And and E. Sidiropoulou, Ind. Eng. Chem. Res., 1997, 36, 1253-1267.

23 R. Darvishi, M. N. Esfahany and R. Bagheri, Ind. Eng. Chem. Res., 2015, 54, 10953-10963.

24 M. W. Allsopp, Pure Appl. Chem., 1981, 53, 449-465.

25 J. Purmová, Effect of the Modification of the Polymer-rich Phase Composition on the Formation of Structural Defects in Radical Suspension PVC, University of Groningen, 2007.

26 G. Palma, M. Tavan and M. Carenza, J. Polym. Sci., Polym. Phys. Ed., 1983, 21, 251-262.

27 P. V. Smallwood, Macromol. Symp., 1989, 29, 1-19.

28 M. Zerfa and B. W. Brooks, J. Appl. Polym. Sci., 1996, 60, 2077-2086.

29 E. F. Márquez and L. L. Lagos, AIChE J., 2004, 50, 3184-3194.

30 B. E. Törnell and J. M. Uustalu, Polymer, 1986, 27, 250-252. 